\title{
A Tissue-Specific Role for Nlrp3 in Tubular Epithelial Repair after Renal Ischemia/Reperfusion
}

Pieter J. Bakker, ${ }^{\star}$ Loes M. Butter, ${ }^{\star}$ Nike Claessen, ${ }^{\star}$ Gwendoline J.D. Teske, ${ }^{\star}$ Fayyaz S. Sutterwala, ${ }^{\dagger}$ Sandrine Florquin, ${ }^{\star \ddagger}$ and Jaklien C. Leemans*

From the Department of Pathology,* Academic Medical Center, Amsterdam, the Netherlands; the Inflammation Program, ${ }^{\dagger}$ University of Iowa, Iowa City, Iowa; and the Department of Pathology, ${ }^{\ddagger}$ Radboud University Nijmegen Medical Center, Nijmegen, the Netherlands

\author{
Accepted for publication \\ April 8, 2014. \\ Address correspondence to \\ Pieter J. Bakker, M.Sc., \\ Department of Pathology, Aca- \\ demic Medical Center, Room \\ L2-112, Meibergdreef 9, 1105 \\ AZ Amsterdam, the Nether- \\ lands. E-mail: j.bakker@amc \\ uva.nl.
}

\begin{abstract}
Ischemia/reperfusion injury is a major cause of acute kidney injury. Improving renal repair would represent a therapeutic strategy to prevent renal dysfunction. The innate immune receptor Nlrp3 is involved in tissue injury, inflammation, and fibrosis; however, its role in repair after ischemia/reperfusion is unknown. We address the role of Nlrp3 in the repair phase of renal ischemia/reperfusion and investigate the relative contribution of leukocyte- versus renal-associated Nlrp3 by studying bone marrow chimeric mice. We found that Nlrp3 expression was most profound during the repair phase. Although Nlrp3 expression was primarily expressed by leukocytes, both leukocyte- and renal-associated Nlrp3 was detrimental to renal function after ischemia/reperfusion. The Nlrp3-dependent cytokine IL-1 $\beta$ remained unchanged in kidneys of all mice. Leukocyte-associated Nlrp3 negatively affected tubular apoptosis in mice that lacked Nlrp3 expression on leukocytes, which correlated with reduced macrophage influx. Nlrp3-deficient (Nlrp3KO) mice with wild-type bone marrow showed an improved repair response, as seen by a profound increase in proliferating tubular epithelium, which coincided with increased hepatocyte growth factor expression. In addition, NLrp3KO tubular epithelial cells had an increased repair response in vitro, as seen by an increased ability of an epithelial monolayer to restore its structural integrity. In conclusion, Nlrp3 shows a tissue-specific role in which leukocyte-associated Nlrp3 is associated with tubular apoptosis, whereas renal-associated Nlrp3 impaired wound healing. (Am J Pathol 2014, 184: 2013-2022; http://dx.doi.org/10.1016/j.ajpath.2014.04.005)
\end{abstract}

Ischemia/reperfusion (IR) injury is a major cause of acute kidney injury ${ }^{1}$ and increases the risk of developing chronic kidney disease (CKD). ${ }^{2}$ After injury, wounded tissue organizes an efficient response that aims to combat infections, clear cell debris, re-establish cell number, and reorganize tissue architecture. First, necrotic tissue releases dangerassociated molecular patterns, such as high-mobility group box $-1^{3}$ or mitochondrial DNA, ${ }^{4}$ which leads to chemokine secretion $^{5}$ and a subsequent influx of leukocytes. Second, neutrophils and macrophages clear cellular debris but also increase renal damage because depletion of neutrophils ${ }^{6}$ or macrophages within 48 hours of IR will reduce renal damage. ${ }^{7}$ At approximately 72 hours of reperfusion, the inflammatory phase transforms into the repair phase and is characterized by surviving tubular epithelial cells (TECs) that dedifferentiate, migrate, and proliferate to restore renal function. ${ }^{8}$
Previously, we have shown that Toll-like receptor (TLR) 2 and TLR4 play a detrimental role after acute renal IR injury. ${ }^{9-11}$ In addition, TLR2 appeared also pivotal in mediating tubular repair in vitro after cisplatin-induced injury, ${ }^{12}$ indicating a dual role for TLR2. The cytosolic innate immune receptor Nlrp3 is able to sense cellular damage ${ }^{13}$ and mediates renal inflammation and pathological characteristics after $\mathrm{IR}^{14-16}$ or nephrocalcinosis. ${ }^{17}$ Next to the detrimental role of Nlrp3 in different renal disease models and consistent with the dual role of TLR2, Nlrp3 was shown to protect against loss of colonic epithelial integrity. ${ }^{18} \mathrm{We}$, therefore, speculate that Nlrp3, which contributes to sterile renal inflammation during acute renal IR injury, might also drive subsequent tubular repair.

\footnotetext{
Supported by Dutch Kidney Foundation grant C06.6023 (P.J.B.) and Dutch Organization for Scientific Research grant 91712386 (L.M.B. and J.C.L.).

Disclosures: None declared.
} 
To test this hypothesis, we investigated the role of leukocyte- versus renal-associated Nlrp3 with respect to tissue repair after renal IR. We observed that both renal- and leukocyte-associated Nlrp3s are detrimental to renal function after renal IR injury; however, this is through different mechanisms. Leukocyte-associated Nlrp3 is related to increased tubular epithelial apoptosis, whereas renal-associated Nlrp3 impairs the tubular epithelial repair response. Our data suggest Nlrp3 as a negative regulator of resident tubular cell proliferation in addition to its detrimental role in renal fibrosis and inflammation. ${ }^{14,19}$

\section{Materials and Methods}

\section{Mice}

Pathogen-free, 8- to 12-week-old, male, wild-type (WT) C57BL/6 mice were purchased from Charles River Laboratories (Cologne, Germany). The generation of Nlrp3-deficient mice was described previously. ${ }^{20}$ Nlrp3-deficient mice were backcrossed onto a C57BL/6 genetic background for nine generations and bred in the animal facility of the Academic Medical Center (Amsterdam, the Netherlands). The CD45.1-positive allotype WT mice used to generate chimeric mice were purchased from Charles River Laboratories (B6.SJL-PtprcaPepcb/BoyCrl strain). Only age- and sexmatched mice were used in all experiments. The Animal Care and Use Committee of the University of Amsterdam (Amsterdam) approved all experiments.

\section{Bone Marrow Transplantation}

Transplantation was performed as described previously. ${ }^{10}$ Briefly, the tibia, femur, and spleen of WT CD45.1 or Nlrp3KO CD45.2 donor mice were harvested. Bone marrow was isolated from tibia and femur. Subsequently, a mixture of bone marrow and splenocytes was i.v. injected into lethally irradiated (two pulses of $4.5 \mathrm{~Gy}$ ) WT CD45.1 or Nlrp3KO $C D 45.2$ recipient mice. Recipient mice received neomycincontaining water 1 week before transplantation up to 4 weeks after transplantation. Six weeks after transplantation, renal ischemia was applied, followed by 5 days of reperfusion. Blood collected via heart puncture before centrifugation was used to assess bone marrow transplantation efficiency. Erythrocytes were lysed, and cells were stained using anti-CD45.1 phosphatidylethanolamine (BD Pharmingen, Breda, the Netherlands) and anti-CD45.2 fluorescein isothiocyanate (BD Pharmingen). Expression was assessed using flow cytometry. The cutoff for a successful transplantation was set at $>75 \%$ positivity.

\section{Renal IR}

Renal IR injury was induced as described previously. ${ }^{9}$ Briefly, both renal pedicles were clamped for 35 minutes using microaneurysm clamps through a midline abdominal incision under general anesthesia $[1.25 \mathrm{mg} / \mathrm{mL}$ midazolam (Actavis, Dublin, Ireland), $0.08 \mathrm{mg} / \mathrm{mL}$ fentanyl citrate, and $2.5 \mathrm{mg} / \mathrm{mL}$ fluanisone (VetaPharma Limited, Leeds, UK)]. After clamp removal, kidneys were inspected for restoration of blood flow. The abdomen was closed in two layers, and all mice received a s.c. injection of $0.1 \mathrm{mg} / \mathrm{kg}$ buprenorphine (Temgesic; Schering-Plough, Amstelveen, the Netherlands). To maintain fluid balance, mice were supplemented with a few drops of sterile $0.9 \% \mathrm{NaCl}$ i.p. before closing the abdomen. Mice were sacrificed 5 days after surgery. Mice were sacrificed and blood was collected by heart puncture, and plasma was collected by centrifugation. Kidneys were snap frozen in liquid nitrogen or formalin fixed, followed by paraffin embedding.

\section{Biochemical Analysis}

Plasma urea and creatinine were measured using standardized clinical diagnostic protocols of the Academic Medical Center.

\section{Quantitative PCR}

Total RNA was isolated from frozen sections using TRIzol (Life Technologies, Bleiswijk, the Netherlands), following the recommended manufacturer's protocol. Complementary DNA was made by ligation of oligo-dT primers and subsequent polymerization using Taq DNA polymerase (Invitrogen, Bleiswijk, the Netherlands). Finally, cDNA was quantified in real time on a Roche LightCycler 480 (Roche Diagnostics, Woerden, the Netherlands) using LightCycler 480 DNA SYBR Green (Roche Diagnostics). To quantify gene expression, cyclophilin $\mathrm{G}$ was used as an endogenous reference. The following primer sequences were used: Nlrp3, 5'-CCACAGTGTAACTTGCAGAAGC-3' (forward) and 5'-GGTGTGTGAAGTTCTGGTTGG-3' (reverse); kidney injury molecule (KIM)-1, 5' -TGGTTGCCTTCCGTGTCTCT-3' (forward) and 5'-TCAGCTCGGGAATGCACAA-3' (reverse); neutrophil gelatinase-associated lipocalin (NGAL), 5'-GCCTCAAGGACGACAACATC-3' (forward) and 5'-CTGAACCATTGGGTCTCTGC-3' (reverse); and hepatocyte growth factor (HGF), 5'-CCCAAGTGTGACGTGTCAAGTGG-3' (forward) and 5'-TGGCTCCCAGAACATATGACGGTGT-3' (reverse).

\section{Cytokine Expression}

Snap-frozen kidneys were lysed using Greenberg Lysis Buffer ( $75 \mathrm{mmol} / \mathrm{L} \mathrm{NaCl}, 7.5 \mathrm{mmol} / \mathrm{L}$ Tris, $0.5 \mathrm{mmol} / \mathrm{L} \mathrm{MgCl} \cdot \mathrm{H}_{2} \mathrm{O}$, $0.5 \mathrm{mmol} / \mathrm{L} \mathrm{CaCl}_{2}$, and $0.5 \%$ Triton X-100), supplemented with protease inhibitor cocktail (Sigma-Aldrich, Zwijndrecht, the Netherlands), according to weight of the kidney. Subsequently, homogenates were used to determine cytokine levels for IL-1 $\beta$, IL-6, keratinocyte chemoattractant (KC), and monocyte chemoattractant protein (MCP)-1, according to the manufacturer's instructions (R\&D Systems, Abingdon, UK). 


\section{Immunohistochemistry}

For PAS-diastase, paraffin-embedded tissue sections were deparaffinized and incubated with $0.25 \%$ amylase solution (Sigma-Aldrich). Subsequently, slides were incubated with $1 \%$ periodic acid (Merck, Amsterdam, the Netherlands), followed by Schiff reagent (Merck). Counterstaining was performed using hematoxylin (Sigma-Aldrich). For cleaved caspase 3, Ki-67, and F4/80, sections were boiled in 0.01 $\mathrm{mol} / \mathrm{L} \mathrm{pH} 6.0$ citrate buffer and subsequently exposed to a rabbit anti-mouse cleaved caspase 3 (Asp175; Bioke, Leiden, the Netherlands), rabbit anti-mouse Ki-67 (Neomarkers, Waltham, MA), or rat IgG2b anti-mouse F4/80 (Serotec, Oxford, UK), respectively. Staining was visualized using a power anti-rabbit poly horseradish peroxidase (Dako, Heverlee, Belgium) and, in addition, a rabbit anti-rat antibody (Dako) in case of F4/80.

\section{Quantification of Immunohistochemistry}

Tubular necrosis is characterized by desquamation of necrotic tubular cells, denudation of tubular basement membrane, and formation of cellular casts. Necrotic cells are characterized by loss of distinguishable nucleus, swelling of the cytoplasm, and loss of brush border. Tubular necrosis was assessed on PAS-diastase sections by a renal pathologist (S.F.) using a semiquantitative score, as follows: $0,0 \% ; 1,1 \%$ to $10 \% ; 2,11 \%$ to $25 \% ; 3,26 \%$ to $50 \% ; 4$, $51 \%$ to $75 \%$; and $5,76 \%$ to $100 \%$ per high-powered field ( $\times 400$ magnification). Cleaved caspase-3- and Ki-67positive TECs were counted in 10 randomly selected highpowered fields $(\times 400$ magnification). F4/80 staining was quantified using a Dotpro Slidescanner (Tokyo, Japan) to obtain digital images, followed by ImageJ software version 1.46r (NIH, Bethesda, MD) to quantify the number of positive pixels.

\section{Immunoprecipitation of IL-1 $\beta$}

To detect IL-1 $\beta$, we immunoprecipitated $1 \mathrm{mg}$ of protein derived from snap-frozen kidneys by lysis of renal tissue with isolation buffer $(20 \mathrm{mmol} / \mathrm{L}$ Tris- $\mathrm{HCl}, \mathrm{pH} \mathrm{7.4,} 135$ $\mathrm{mmol} / \mathrm{L} \mathrm{NaCl}, 1.5 \mathrm{mmol} / \mathrm{L} \mathrm{MgCl} 2,10 \%$ glycerol, and $1 \%$ Triton X-100). Subsequently, all IL-1 $\beta$ was isolated using an anti-IL-1 $\beta$ rat IgG1 monoclonal antibody (MAB401; R\&D Systems), followed by precipitation using Protein G-Sepharose beads (Biovision, Milpitas, CA). Subsequently, all precipitated IL-1 $\beta$ was visualized with Western blot analysis using an anti-IL-1 $\beta$ polyclonal goat IgG (BAF401; R\&D Systems) and streptavidin-poly horseradish peroxidase (Sanquin Blood Supply, Amsterdam, the Netherlands). As a positive control for immunoprecipitation or Western blot analysis, we added $100 \mathrm{pg}$ of recombinant IL-1 $\beta$ (R\&D Systems) to $1 \mathrm{mg}$ of protein or $50 \mathrm{pg}$ of recombinant IL-1 $\beta$ (R\&D Systems), respectively. A band at $18 \mathrm{kDa}$ was interpreted as active IL-1 $\beta$.

\section{In Vitro Hypoxia Assay}

Proximal TECs were isolated from kidneys, as described previously, ${ }^{9}$ and cultured using HK2 medium supplemented with $10 \%$ fetal calf serum, $2 \mathrm{mmol} / \mathrm{L}$ L-glutamine, 100 $\mathrm{IU} / \mathrm{mL}$ penicillin, and $100 \mu \mathrm{g} / \mathrm{mL}$ streptomycin (Invitrogen) until cells became $70 \%$ to $80 \%$ confluent. Hypoxia was induced after a protocol described previously. ${ }^{21}$ Ringer ischemia solution $\left(4.5 \mathrm{mmol} / \mathrm{L} \mathrm{NaHCO}_{3}, 0.8 \mathrm{mmol} / \mathrm{L}\right.$ $\mathrm{Na}_{2} \mathrm{HPO}_{4}, 0.2 \mathrm{mmol} / \mathrm{L} \mathrm{NaH}_{2} \mathrm{PO}_{4}, 106.0 \mathrm{mmol} / \mathrm{L} \mathrm{NaCl}, 5.4$ $\mathrm{mmol} / \mathrm{L} \mathrm{KCl}, 1.2 \mathrm{mmol} / \mathrm{L} \mathrm{CaCl}_{2}, 0.8 \mathrm{mmol} / \mathrm{L} \mathrm{MgCl}_{2}$, and $20 \mathrm{mmol} / \mathrm{L}$ morpholinoethanesulfonic acid, $\mathrm{pH}$ 6.6) was made anoxic by mixing $100 \% \mathrm{~N}_{2}$ gas with Ringer ischemia solution for 5 minutes. Subsequently, HK2 medium was replaced by Ringer ischemia solution, and cells were transferred to a hypoxic chamber, which was flushed for 1 minute with $100 \% \mathrm{~N}_{2}$ gas. Subsequently, cells were incubated for 4 hours at $37^{\circ} \mathrm{C}$.

\section{In Vitro Wound Healing Assay}

Proximal TECs were cultured using HK2 medium supplemented with $10 \%$ fetal calf serum, $2 \mathrm{mmol} / \mathrm{L}$ L-glutamine, 100 $\mathrm{IU} / \mathrm{mL}$ penicillin, and $100 \mu \mathrm{g} / \mathrm{mL}$ streptomycin (Invitrogen) for 8 days. Subsequently, cells were harvested and seeded at $2 \times 10^{5}$ cells $/ \mathrm{mL}$ and cultured for an additional 2 days to obtain a confluent epithelial monolayer. Finally, a scratch was made using a p1000 pipette tip, and cells were monitored for the following 72 hours using in-house designed software and a phase-contrast microscope. Recovery was assessed by measuring nonrecovered wound area at marked points where the scratched area at $\mathrm{t}=0$ was set at $100 \%$.

\section{Statistical Analysis}

All data are presented as means \pm SEM. Statistical analyses were performed using the nonparametric Kruskal-Wallis test with Dunn's correction or the parametric one-way analysis of variance with Bonferroni's multiple comparison test in case of equal variances to test for multiple group comparison. The nonparametric $U$-test was used for two-group comparisons. For all analyses, $P<0.05$ was considered significant.

\section{Results}

\section{Nlrp3 Gene Expression Associates with Renal Repair Phase}

We first investigated Nlrp3 gene expression in ischemic kidneys after 1, 5, and 10 days of reperfusion. Nlrp3 gene expression peaked after 5 days of reperfusion, with a fivefold increase compared with sham levels (Figure 1A). Similar to Nlrp3 expression in macrophages on lipopolysaccharide (LPS) stimulation, ${ }^{20}$ TECs up-regulated Nlrp 3 gene expression after hypoxia or on LPS stimulation (Figure 1B). To 
determine the role of renal- versus leukocyte-associated Nlrp3 during the repair phase (ie, after 5 days of reperfusion), we generated bone marrow chimeric mice made by reconstituting sublethally irradiated WT or Nlrp3KO mice with bone marrow taken from either WT or Nlrp $3 \mathrm{KO}$ mice. After 5 days of reperfusion, we found that Nlrp3 is primarily expressed by infiltrating leukocytes, as seen by almost similar Nlrp3 gene expression in Nlrp3KO mice transplanted with WT bone marrow [WT $\rightarrow$ knockout (KO)] compared with WT mice transplanted with WT bone marrow $(\mathrm{WT} \rightarrow \mathrm{WT}$ ) (Figure 1C). Next, we found renal parenchymal Nlrp3 expression, as seen by an increased expression in WT mice transplanted with Nlrp3KO bone marrow $(\mathrm{KO} \rightarrow \mathrm{WT})$ compared with Nlrp3KO mice transplanted with Nlrp3KO bone marrow $(\mathrm{KO} \rightarrow \mathrm{KO}$ ) (Figure $1 \mathrm{C})$. In conclusion, Nlrp3 is expressed both by leukocytes and renal parenchymal cells after 5 days of reperfusion, although leukocyte-associated Nlrp 3 gene expression is dominant.

\section{Both Renal- and Leukocyte-Associated Nlrp3 Mediates Renal Dysfunction}

Because Nlrp3 is expressed in both compartments, we investigated the role of leukocyte- versus renal-associated Nlrp3 expression on renal function after IR. Both renal-and leukocyte-associated Nlrp3 mediated loss of renal function, as seen by decreased levels of plasma urea (Figure 2A) or plasma creatinine (Figure 2B) in $\mathrm{WT} \rightarrow \mathrm{KO}, \mathrm{KO} \rightarrow \mathrm{WT}$, and $\mathrm{KO} \rightarrow \mathrm{KO}$ mice compared with WT $\rightarrow$ WT mice after 5 days of reperfusion. Bone marrow chimeras were also subjected to ischemia, followed by 1 day of reperfusion, to investigate the early contribution of renal versus leukocyte Nlrp3 on renal function. Herein, we observed that only renal Nlrp3 mediates renal dysfunction on the basis of plasma creatinine levels (Supplemental Figure S1, A and B). We next investigated the renal damage markers KIM- ${ }^{22}$ and $\mathrm{NGAL}^{23}$ to investigate proximal versus distal damage. Both chimeras showed an expression pattern similar to plasma urea and creatinine with respect to KIM-1 (Figure 2C) and NGAL (Figure 2D), confirming that both leukocyte- and renalassociated Nlrp3s are detrimental after renal IR injury and indicating that distinct Nlrp3 expression has no differential effect on proximal versus distal tubular damage.

\section{Nlrp3 Does Not Lead to a Differential Proinflammatory Cytokine Production}

To determine whether the beneficial effect of Nlrp3 deficiency after renal IR injury was due to inhibition of cytokines and chemokines, we additionally investigated renal levels of KC, MCP-1, IL-1 $\beta$, and IL-6. The renal chemokines $\mathrm{KC}$ and MCP-1 remained unchanged in $\mathrm{WT} \rightarrow \mathrm{KO}$, $\mathrm{KO} \rightarrow \mathrm{KO}$ mice, but were decreased in $\mathrm{KO} \rightarrow \mathrm{WT}$ mice compared with WT $\rightarrow$ WT animals (Figure 3, A and B). We observed that the proinflammatory cytokines IL- $1 \beta$ and IL-6 were not differentially expressed in kidneys of chimeras after 5 days of reperfusion (Figure 3, C and D). To differentiate proactive versus active $\mathrm{IL}-1 \beta$, we performed immunoprecipitation, followed by blotting of kidney homogenates for active IL-1 $\beta$. Although levels are overall low, we observed that WT $\rightarrow \mathrm{KO}$ mice had an increase in active $\mathrm{IL}-1 \beta$ versus $\mathrm{WT} \rightarrow \mathrm{WT}$ mice, whereas $\mathrm{KO} \rightarrow \mathrm{KO}$ and $\mathrm{KO} \rightarrow$ WT mice show a heterogeneous amount of active IL$1 \beta$ in homogenates (Figure 3E).

\section{Leukocyte-Associated Nlrp3 Mediates Tubular Apoptosis}

Because the Nlrp3 inflammasome is also implicated in the induction of cell death, ${ }^{15,24}$ we next investigated tubular necrosis and apoptosis as underlying causes for renal dysfunction using PAS-diastase- and cleaved caspase-3-stained sections (Figure 4, A and C). $\mathrm{KO} \rightarrow$ WT mice showed a strong reduction in the amount of tubular necrosis (Figure 4B), which was consistent with improved renal function. Unexpectedly, we observed a similar degree of tubular necrosis in WT $\rightarrow \mathrm{KO}$ mice compared with WT $\rightarrow$ WT mice, which did not correlate with improved renal function, suggesting a different underlying cause (Figure 4B). Cleaved caspase-3 staining of renal sections (Figure 4C) showed a pattern similar to necrosis; however, herein, all mice that lacked Nlrp3 expression on leukocytes showed a reduction in apoptotic TECs (Figure 4D).
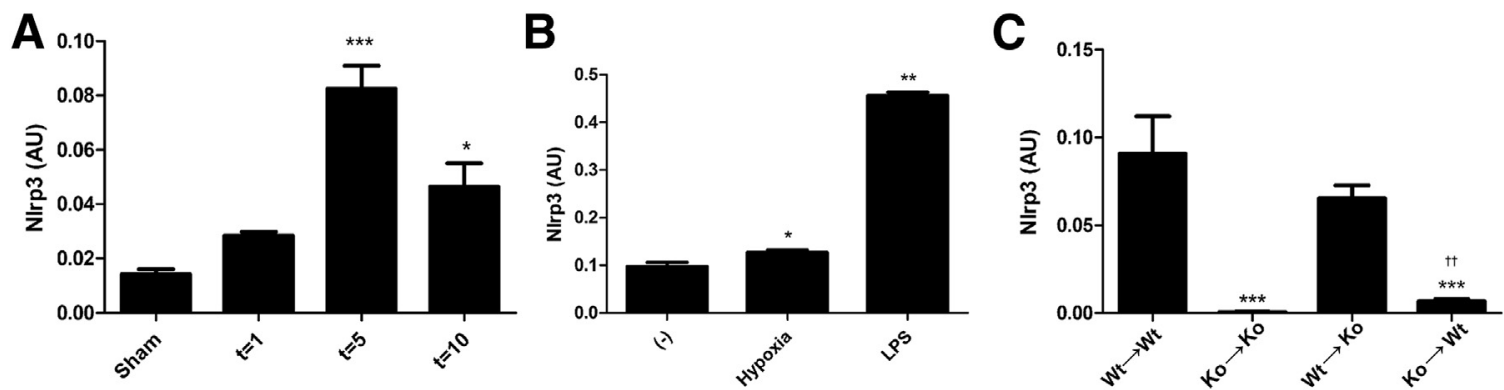

Figure 1 Nlrp3 gene expression after IR. Nlrp3 gene expression after ischemia in WT mice that were sham operated on or after 1, 5, or 10 days of reperfusion (A), renal tubular epithelium ( $n=3$ to 7 ) after hypoxia ( 4 hours) or stimulated with LPS (18 hours) (B), or WT/Nlrp3K0 chimeras after 5 days of reperfusion (C). Results are expressed as means $\pm \operatorname{SEM}(n=7$ to 11 animals per group). Statistics were performed using one-way analysis of variance. ${ }^{*} P<0.05,{ }^{* *} P<0.01$, and ${ }^{* *} P<0.001$ versus sham or WT $\rightarrow$ WT mice (Dunnett's multiple-comparison test); ${ }^{{ }^{\dagger \dagger}} P<0.01$ versus $\mathrm{K} 0 \rightarrow \mathrm{K} 0$ mice $(U$-test). 

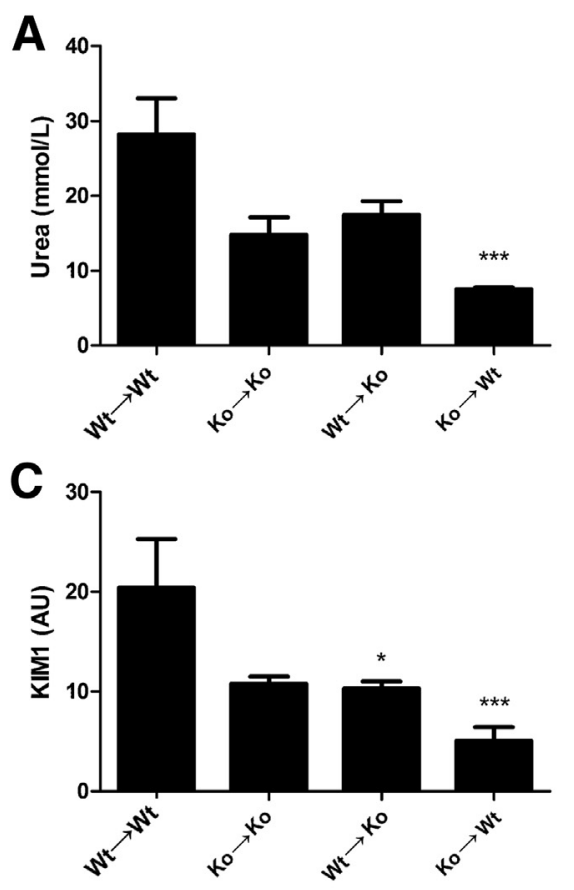
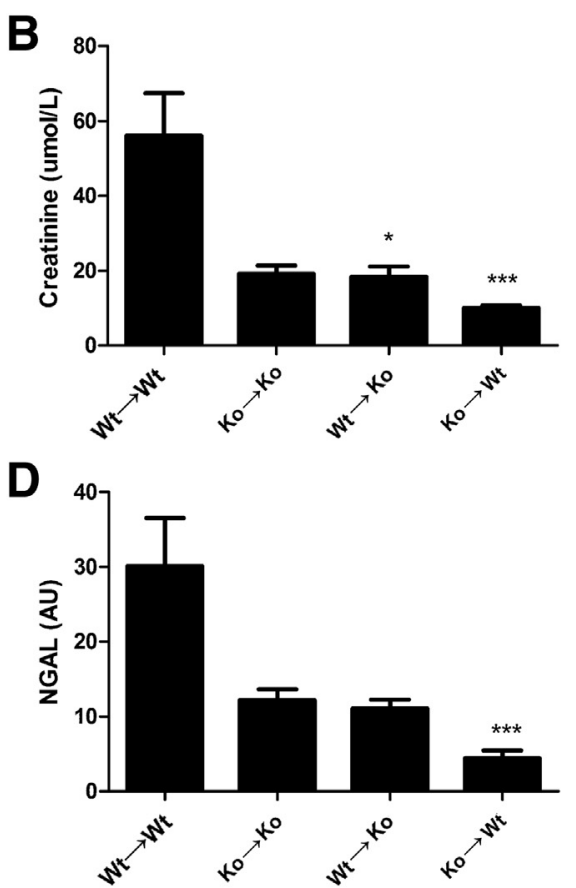

Figure 2 Both renal- and leukocyte-associated Nlrp3 deficiency protects against renal dysfunction. Renal function was assessed after 5 days of reperfusion using the markers plasma urea $(\mathbf{A})$ and creatinine (B). Proximal and distal tubular damage was investigated using the proximal marker KIM-1 (C) and distal marker NGAL (D). Results are expressed as means \pm SEM ( $n=7$ to 11 animals per group). Statistics were performed using the KruskalWallis test with Dunn's multiple-comparison test. ${ }^{*} P<0.05,{ }^{* *} P<0.001$ versus WT $\rightarrow$ WT mice.
Macrophage Influx Correlates with Tubular Cell Death

Depletion of macrophages after IR reduces renal damage. ${ }^{25}$ In this light, we examined the presence of macrophages using the macrophage marker F4/80 (Figure 5A). Macrophage numbers were decreased in $\mathrm{KO} \rightarrow \mathrm{KO}$ and $\mathrm{KO} \rightarrow \mathrm{WT}$ groups compared with WT $\rightarrow \mathrm{WT}$ mice (Figure 5B). $\mathrm{WT} \rightarrow \mathrm{KO}$ mice had similar numbers of macrophages as WT $\rightarrow$ WT mice. Finally, we correlated macrophage influx to tubular necrosis (Figure 5C) and apoptosis (Figure 5D) and observed that macrophage influx was highly significantly related to tubular cell death.

Increased Tubular Epithelial Proliferation in Nlrp3KO Mice Transplanted with WT Bone Marrow

Because the amount of tubular cell death and renal inflammation in WT $\rightarrow \mathrm{KO}$ mice did not correlate with improved
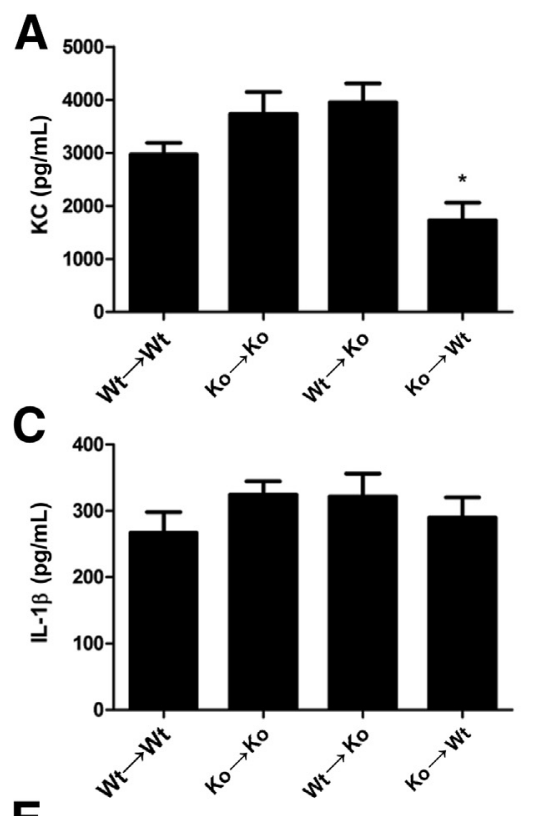

E

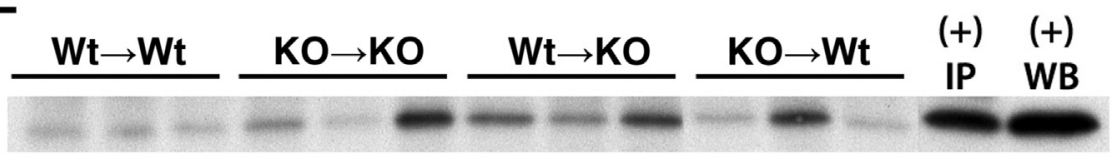

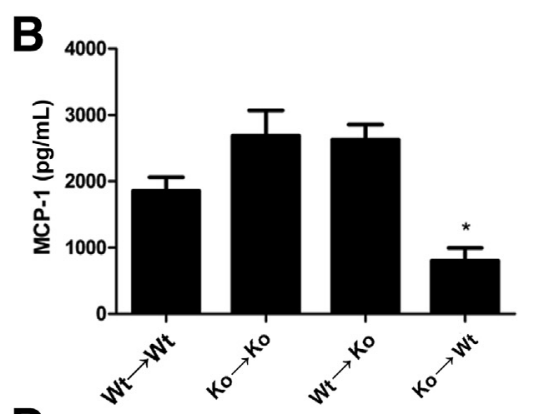

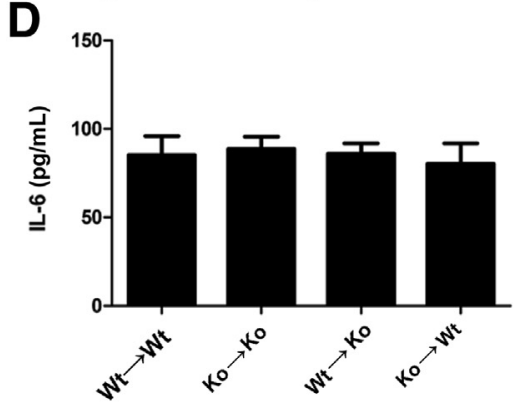

Figure 3 Nlrp3 does not regulate proinflammatory cytokine production. Renal cytokine levels of KC (A), MCP-1 (B), IL-1 $\beta$ (C), and IL-6 (D) were measured. E: Active IL-1 $\beta$ was analyzed by immunoprecipitation and Western blot analysis of three representative mice per group. Results are expressed as means \pm SEM ( $n=7$ to 11 animals per group). Statistics were performed using oneway analysis of variance with Dunnett's multiplecomparison test. ${ }^{\star} P<0.05$ versus WT $\rightarrow$ WT mice. 


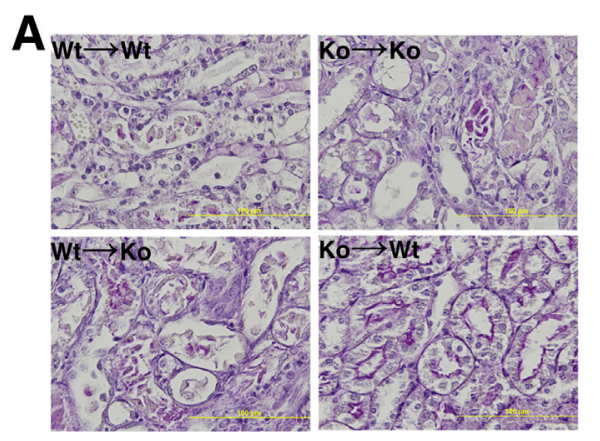

C

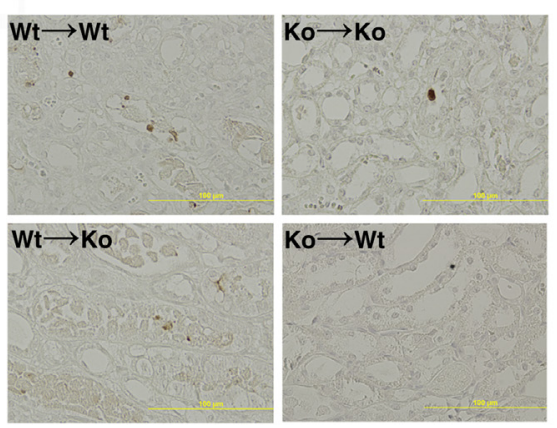

B

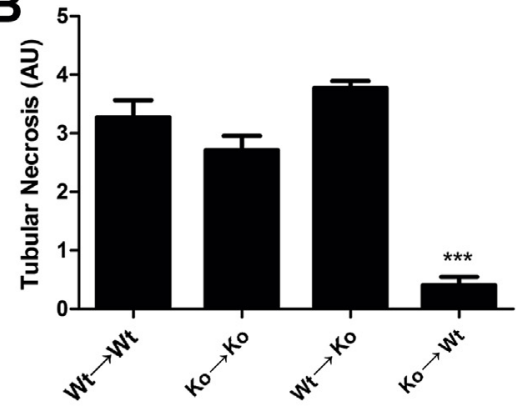

D

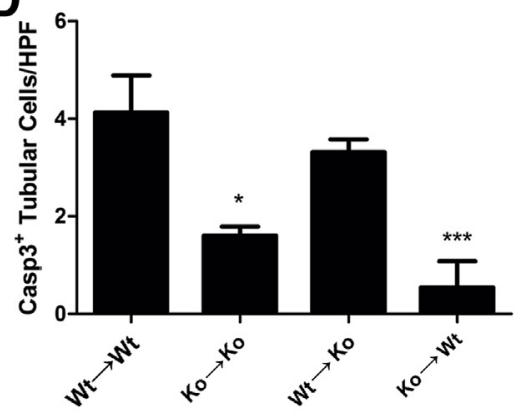

Figure 4 Leukocyte-associated Nlrp3 regulates tubular apoptosis. PAS-diastase-stained renal sections (A) were semiquantitatively scored for tubular necrosis (B). Renal sections were stained for the apoptosis marker cleaved caspase-3 (C), and positive tubular cells were quantified (D). Results are expressed as means \pm SEM ( $n=7$ to 11 animals per group). Statistics were performed using the Kruskal-Wallis test with Dunn's correction. ${ }^{*} P<0.05,{ }^{* * *} P<0.001$ versus $\mathrm{WT} \rightarrow \mathrm{WT}$ mice. $\mathrm{AU}$, arbitrary unit. renal function, we next investigated the tubular repair response using the proliferation marker Ki-67 (Figure 6A). Remarkably, we observed a twofold increase in proliferating TECs in $\mathrm{WT} \rightarrow \mathrm{KO}$ mice, suggesting an improved repair response. Consistent with tubular necrosis, we observed a decrease in $\mathrm{KO} \rightarrow \mathrm{WT}$ mice and a trend in $\mathrm{KO} \rightarrow \mathrm{KO}$ mice compared with $\mathrm{WT} \rightarrow \mathrm{WT}$ mice (Figure 6B). Although growth factors contribute to renal repair, ${ }^{26}$ we did not observe an increase in gene expression of insulin-like growth factor-1, fibroblast growth factor-1, or fibroblast growth factor-7 in $\mathrm{WT} \rightarrow \mathrm{KO}$ mice compared with WT $\rightarrow$ WT mice (data not shown). In contrast, renal HGF gene expression was profoundly up-regulated in $\mathrm{WT} \rightarrow \mathrm{KO}$ mice (Figure 6C).

\section{Nlrp3KO Tubular Epithelium Shows an Increased Repair Response}

In addition to extrinsic factors, such as HGF, we also investigated whether intrinsic differences in repair exist between WT and Nlrp3KO tubular epithelium. By using an in vitro wound healing assay, we observed that Nlrp3KO tubular epithelium had an increased capacity to restore integrity of an
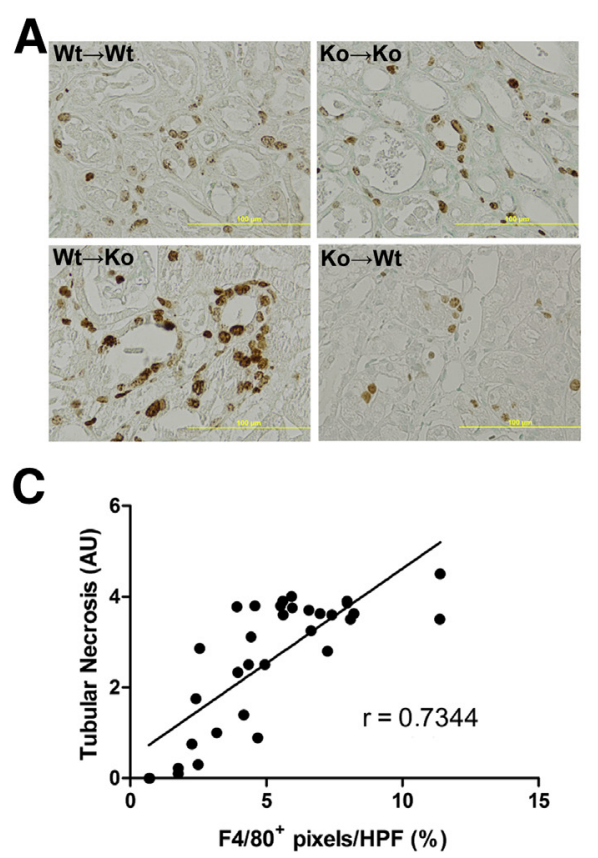

B

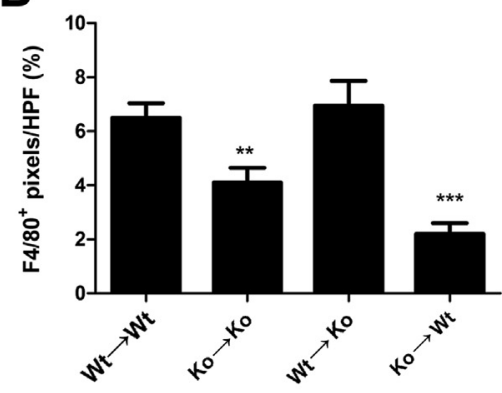

D

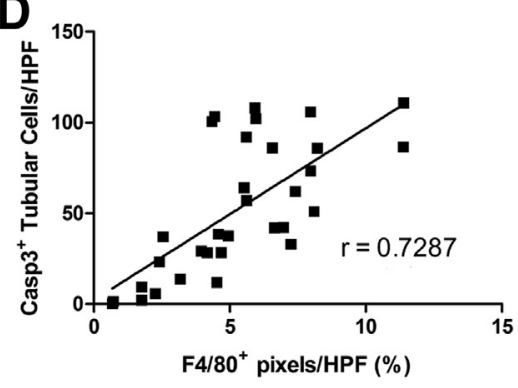

Figure 5 Macrophage accumulation correlates with tubular cell death. Immunohistochemistry of the murine macrophage marker $F / / 80$ (A) and quantification of renal sections (B). Correlation of $\mathrm{F} 4 / 80^{+}$area with semiquantitatively scored necrosis (C) or amount of cleaved caspase- $3^{+}$TECS (D). Results are expressed as means \pm SEM ( $n=7$ to 11 animals per group). Statistics were performed using one-way analysis of variance: ${ }^{* *} P<0.01,{ }^{* * *} P<0.001$ versus WT $\rightarrow$ WT mice (Dunnett's multiple-comparison test; B); $P<$ 0.0001 (Spearman's test for correlation; C and D). $\mathrm{AU}$, arbitrary unit; HPF, high-powered field. 

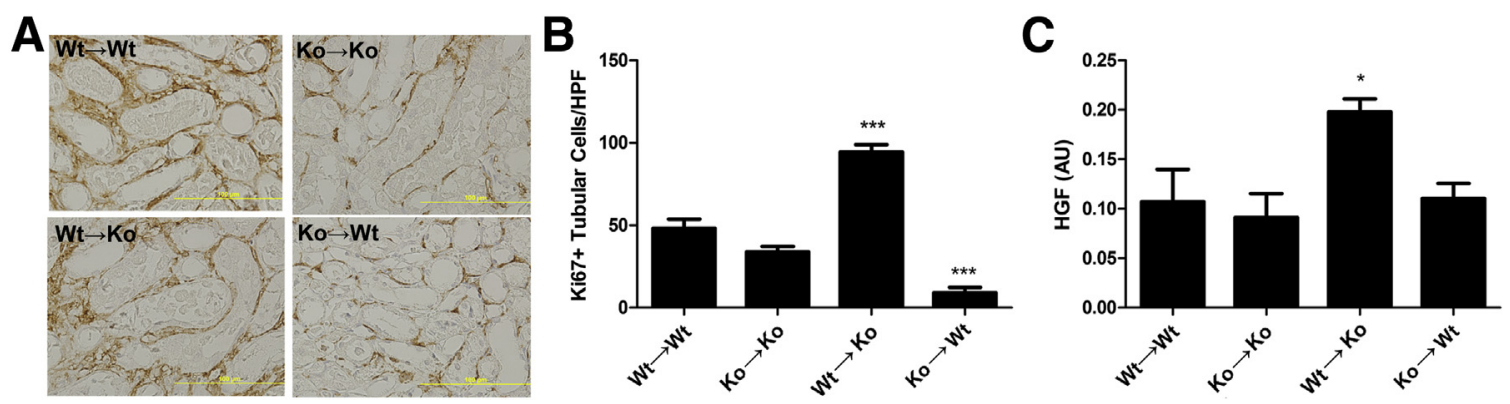

Figure 6 Increased proliferating tubular epithelium in Nlrp3KO mice transplanted with WT bone marrow. Renal sections stained for the proliferation marker Ki-67 (A) and the amount of Ki-67 ${ }^{+}$TECs quantified (B). C: Renal gene expression of HGF. Results are expressed as means \pm SEM ( $n=7$ to 11 animals per group). Statistics were performed using a one-way analysis of variance with Dunnett's correction. ${ }^{*} P<0.05,{ }^{* *} P<0.001$ versus WT $\rightarrow$ WT mice. AU, arbitrary unit; HPF, high-powered field.

epithelial monolayer after an insult (Figure 7A). Time to initiate a repair response was decreased in Nlrp3KO tubular epithelium (Figure 7B). During repair, few cells lost cell-cell contact and migrated as single cells into the damaged area (Supplemental Movie S1 and Supplemental Movie S2). We quantified migrating single cells and observed increased numbers in WT tubular epithelium after 20 hours compared with Nlrp3KO tubular epithelium (Figure 7C). In conclusion, Nlrp3KO tubular epithelium showed a better repair response after an insult, which correlates with the increased proliferation of TECs we found in $\mathrm{WT} \rightarrow \mathrm{KO}$ mice.

\section{Discussion}

Improving repair after renal IR would represent a therapeutic strategy to reduce renal dysfunction. The innate immune receptor Nlrp3 is involved in the induction of renal damage, inflammation, ${ }^{14}$ and fibrosis ${ }^{19}$; however, its role on repair after IR is unknown. Herein, we show that both renal- and leukocyte-associated Nlrp3s mediate loss of renal function, although through different mechanisms. Leukocyteassociated Nlrp3 mediates tubular apoptosis, whereas renalassociated Nlrp3 diminishes the repair response, as seen by more proliferating TECs in vivo and the ability to restore the integrity of an epithelial monolayer in vitro.

Nlrp3 has been shown to be detrimental to renal function after 1 day of reperfusion. ${ }^{14,15}$ However, a role for Nlrp3 at the repair phase of renal IR injury was not investigated. We found that Nlrp3 gene expression (this study) and tubular proliferation ${ }^{10}$ were highest after 5 days of reperfusion, suggesting an important role for Nlrp3 during the repair phase. We, therefore, investigated the role of Nlrp3 on renal repair. We show that TECs express Nlrp3 (this study), as macrophages do. ${ }^{20}$ Current research shows a profibrotic role for Nlrp3 in renal epithelium ${ }^{27}$ and a proinflammatory role in macrophages,${ }^{20}$ indicating cell type-specific effects of Nlrp3 on cellular function. To differentiate leukocyte- from renalassociated Nlrp3, we generated Nlrp3KO chimeras. Leukocyte-associated Nlrp3 gene expression is dominant over renal-associated Nlrp3 after 5 days of reperfusion, which is consistent with the observation that renal function is least impaired in WT mice that lack Nlrp3 expression on leukocytes. This protective effect seems dependent on the stage of disease, because Shigeoka et $\mathrm{al}^{15}$ and we found that leukocyte-associated Nlrp3 expression does not mediate renal dysfunction after 1 day of renal IR (this study). To extend the study of Shigeoka et al, ${ }^{15}$ we generated bone marrow chimeras to investigate the early contribution of renal-associated Nlrp3 to renal dysfunction after 1 day of IR. Herein, renal-associated Nlrp3 mediated renal dysfunction. The observed protection became clearer in the repair phase (day 5), despite low renal-associated Nlrp3 gene expression. Our data indicate that the role of Nlrp3 is dependent on the stage of disease and Nlrp3 has a tissue-specific role.
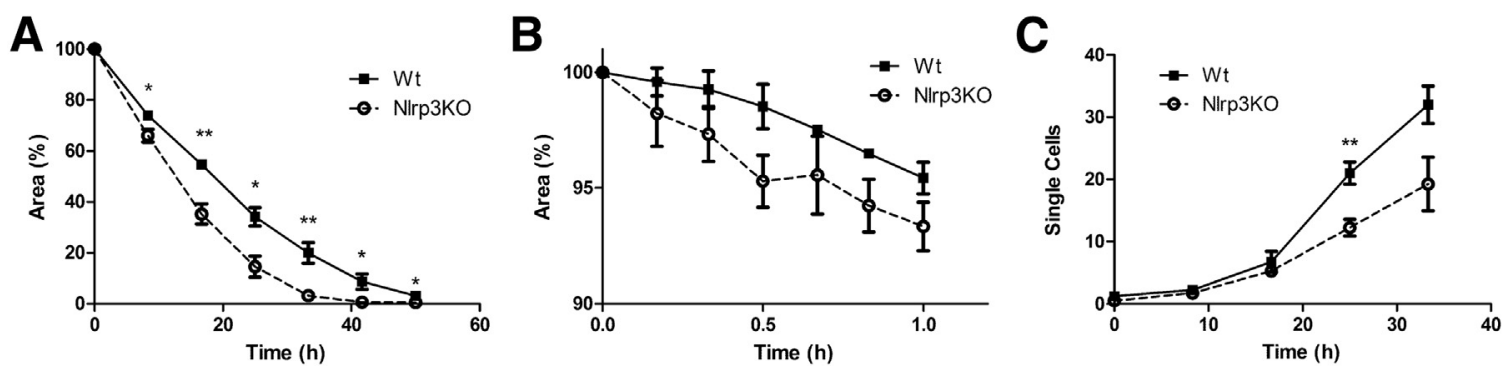

Figure 7 Nlrp3-deficient TECs show an increased regenerative response. An epithelial monolayer was damaged by a scratch. A: The damaged area was measured up to 72 hours after scratch. B: Every 10 minutes, up to 1 hour, the damaged area was measured. C: Single, migratory cells appearing in the damaged area were counted. Results are expressed as means \pm SEM $(n=5)$. Statistics were performed using the $U$-test. ${ }^{\star} P<0.05,{ }^{* * P}<0.01$ versus WT tubular epithelium. 
To understand why Nlrp3 deficiency of leukocytes and renal parenchymal cells results in less impaired renal function in the late phase of renal IR injury, we next analyzed inflammation and tubular cell death. The first consequence of renal IR is tubular cell death, leading to renal dysfunction and cytokine release. ${ }^{28}$ Indeed, we observed that KC and MCP-1 were reduced in WT mice transplanted with Nlrp3KO bone marrow, which also experienced low levels of necrosis. Because Nlrp3 is able to form the Nlrp3 inflammasome and subsequently activate the proinflammatory cytokine IL- $1 \beta$ (canonical pathway), we speculated that a reduction in a proinflammatory response might underlie improved renal function in chimeric mice. However, we did not find differential expression of the proinflammatory cytokines IL- $1 \beta$ or IL-6 nor decreased levels of active IL-1 $\beta$ in Nlrp3 KO mice transplanted with WT or Nlrp3 KO bone marrow. This suggests that alternative mechanisms might be involved in IL-1 $\beta$ maturation on renal IR injury. These results are consistent with other reports showing noncanonical effects of Nlrp3 in the kidney. ${ }^{15,16,27,29}$

Next to cytokines, renal function is influenced by tubular damage and repair. Interestingly, we observed that renal apoptosis was reduced in mice lacking Nlrp3 expression in leukocytes, suggesting that leukocyte-associated Nlrp3 influences tubular epithelial apoptosis and, thereby, renal dysfunction.

One day after reperfusion, the time point at which neutrophil influx peaks, ${ }^{5}$ chimeric mice in which leukocytes were Nlrp3 deficient did not show reduced renal damage ${ }^{15}$ and function (this study). Therefore, we speculated that Nlrp3 in macrophages, of which influx peaks at day $5,{ }^{10}$ rather than neutrophils is important for differences in renal damage in the late phase of renal IR injury. Indeed, macrophages can induce tubular cell apoptosis in models of tubulointerstitial inflammation. Herein, the polarization status of the macrophage and the production of proinflammatory cytokines and cytotoxic products is linked to tubular injury. Consistently, we observed that the level of renal macrophage influx and the amount of apoptotic TECs were both reduced in mice without Nlrp3 on their leukocytes. Additional analysis showed that macrophage influx correlated with the amount of tubular epithelial apoptosis.

Conversely, deficiency of Nlrp3 on renal parenchymal cells did not lead to differences in tubular epithelial apoptosis or necrosis 5 days after renal IR. It was, therefore, of particular interest that, despite similar levels of necrosis and apoptosis, mice deficient for renal parenchymal Nlrp3 with WT bone marrow showed an increased repair response, as seen by a twofold increase in proliferating TECs compared with control WT $\rightarrow$ WT mice. This suggests that renal-associated Nlrp3 is detrimental to renal repair, an observation that was supported by the in vitro wound healing assay. Apparently, Nlrp3 does not drive tubular repair after an insult, as TLR2 does in vitro after cisplatininduced injury. ${ }^{12} \mathrm{We}$ speculate that $\mathrm{KO} \rightarrow \mathrm{KO}$ mice did not show increased numbers of proliferating TECs due to reduced tubular damage needs to be repaired. In addition, we observed reduced levels of proliferating TECs in WT mice, which lacked Nlrp3 expression on leukocytes, which probably reflects the severely reduced level of tubular necrosis that was found in these mice.

Several factors might explain the increased tubular epithelial proliferation in $\mathrm{WT} \rightarrow \mathrm{KO}$ mice. The observed increased renal HGF expression could account for increased tubular epithelial proliferation. Indeed, in a model of $\mathrm{HgCl}_{2-}$ induced renal injury, infused HGF increased tubular epithelial proliferation. ${ }^{30}$ The HGF gene is regulated by IL- $6 .{ }^{31}$ However, IL-6 levels remained unchanged. Nlrp3 regulates IL- $1 \beta,{ }^{32}$ and it was shown that addition of IL- $1 \beta$ enhances HGF secretion of epithelial cells. ${ }^{33}$ Given that active IL-1 $\beta$ was elevated in kidneys of WT $\rightarrow \mathrm{KO}$ mice that show increased proliferation might suggest a role for IL- $1 \beta$ in renal HGF expression. In addition, epithelial Nlrp3 can have intracellular effects on proliferation. Indeed, WT tubular epithelium has a decreased capacity to restore integrity of a monolayer after an insult. Repair in both WT and Nlrp3KO epithelium initiated in a similar manner; however, Nlrp3KO epithelium showed faster closure of the scratched area. The pattern of migration can be used to distinguish fibroblast-like cells as loosely connected migrating cells from epithelial cells that migrate as a sheet. ${ }^{34}$ We observed two patterns of migration in which WT epithelium showed more fibroblastlike cells after 20 hours, whereas Nlrp3KO epithelium maintained epithelial-like behavior. This observation is consistent with articles demonstrating that Nlrp3 enhances renal epithelial-mesenchymal transition ${ }^{27}$ and fibrosis in a model of unilateral ureteral obstruction. ${ }^{19}$ In addition, mice that lack Nlrp3 in renal cells show increased levels of KC and MCP-1, suggesting a prolonged inflammatory phase that could be introduced by a reduced responsiveness to transforming growth factor- $\beta .{ }^{27}$ On the basis of these findings and the results of our study, we speculate that epithelial Nlrp3 is an important regulator of proliferation versus fibrosis during repair. Whether enhanced epithelial proliferation diminishes fibrosis after long-term IR remains to be investigated. However, a recent report showed that Nlrp3 deficiency does not regulate fibrosis after unilateral ureteral obstruction. ${ }^{35}$ More research is warranted to investigate the role of Nlrp3 on ischemia-induced fibrosis.

In conclusion, our research extends the role of Nlrp3 in noncanonical pathways in the diseased kidney. We show herein, using Nlrp3KO chimeras, that leukocyte-associated Nlrp3 negatively affects tubular epithelial apoptosis during the repair phase of renal IR injury. Renal-associated Nlrp3 is detrimental to the repair response, as reflected by enhanced HGF levels, an increased amount of proliferating TECs, and an enhanced ability of epithelium to restore structural integrity in the absence of Nlrp3. Because acute kidney injury is associated with increased CKD incidence, ${ }^{36-39}$ we speculate that increasing renal repair after IR through modulation of renal Nlrp3 might offer therapeutic potential in reducing IR-induced CKD. 


\section{Acknowledgments}

We thank Jan Stap for assistance with the in vitro scratch assay and Onno de Boer for help with digitalization of renal sections.

\section{Supplemental Data}

Supplemental material for this article can be found at http://dx.doi.org/10.1016/j.ajpath.2014.04.005.

\section{References}

1. Thadhani R, Pascual M, Bonventre JV: Acute renal failure. N Engl J Med 1996, 334:1448-1460

2. Ko GJ, Grigoryev DN, Linfert D, Jang HR, Watkins T, Cheadle C, Racusen L, Rabb H: Transcriptional analysis of kidneys during repair from AKI reveals possible roles for NGAL and KIM-1 as biomarkers of AKI-to-CKD transition. Am J Physiol Renal Physiol 2010, 298: F1472-F1483

3. Wu H, Ma J, Wang P, Corpuz TM, Panchapakesan U, Wyburn KR, Chadban SJ: HMGB1 contributes to kidney ischemia reperfusion injury. J Am Soc Nephrol 2010, 21:1878-1890

4. Zhang Q, Raoof M, Chen Y, Sumi Y, Sursal T, Junger W, Brohi K, Itagaki K, Hauser CJ: Circulating mitochondrial DAMPs cause inflammatory responses to injury. Nature 2010, 464:104-107

5. Stroo I, Stokman G, Teske GJ, Raven A, Butter LM, Florquin S, Leemans JC: Chemokine expression in renal ischemia/reperfusion injury is most profound during the reparative phase. Int Immunol 2010, 22:433-442

6. Grenz A, Kim JH, Bauerle JD, Tak E, Eltzschig HK, Clambey ET: Adora2b adenosine receptor signaling protects during acute kidney injury via inhibition of neutrophil-dependent TNF- $\alpha$ release. J Immunol 2012, 189:4566-4573

7. Lee S, Huen S, Nishio H, Nishio S, Lee HK, Choi BS, Ruhrberg C, Cantley LG: Distinct macrophage phenotypes contribute to kidney injury and repair. J Am Soc Nephrol 2011, 22:317-326

8. Bonventre JV: Dedifferentiation and proliferation of surviving epithelial cells in acute renal failure. J Am Soc Nephrol 2003, 14(Suppl 1):S55-S61

9. Pulskens WP, Teske GJ, Butter LM, Roelofs JJ, Van Der Poll T, Florquin S, Leemans JC: Toll-like receptor-4 coordinates the innate immune response of the kidney to renal ischemia/reperfusion injury. PLoS One 2008, 3:9

10. Leemans JC, Stokman G, Claessen N, Rouschop KM, Teske GJ, Kirschning CJ, Akira S, Van Der Poll T, Weening JJ, Florquin S: Renal-associated TLR2 mediates ischemia/reperfusion injury in the kidney. J Clin Invest 2005, 115:2894-2903

11. Wu H, Chen G, Wyburn KR, Yin J, Bertolino P, Eris JM, Alexander SI, Sharland AF, Chadban SJ: TLR4 activation mediates kidney ischemia/reperfusion injury. J Clin Invest 2007, 117: 2847-2859

12. Sallustio F, Costantino V, Cox SN, Loverre A, Divella C, Rizzi M, Schena FP: Human renal stem/progenitor cells repair tubular epithelial cell injury through TLR2-driven inhibin-A and microvesicle-shuttled decorin. Kidney Int 2013, 83:392-403

13. Leemans JC, Cassel SL, Sutterwala FS: Sensing damage by the NLRP3 inflammasome. Immunol Rev 2011, 243:152-162

14. Iyer SS, Pulskens WP, Sadler JJ, Butter LM, Teske GJ, Ulland TK, Eisenbarth SC, Florquin S, Flavell RA, Leemans JC, Sutterwala FS: Necrotic cells trigger a sterile inflammatory response through the Nlrp3 inflammasome. Proc Natl Acad Sci U S A 2009, 106: 20388-20393
15. Shigeoka AA, Mueller JL, Kambo A, Mathison JC, King AJ, Hall WF, Correia Jda S, Ulevitch RJ, Hoffman HM, McKay DB: An inflammasome-independent role for epithelial-expressed Nlrp3 in renal ischemia-reperfusion injury. J Immunol 2010, 185:6277-6285

16. Bakker PJ, Butter LM, Kors L, Teske GJ, Aten J, Sutterwala FS, Florquin S, Leemans JC: Nlrp3 is a key modulator of diet-induced nephropathy and renal cholesterol accumulation. Kidney Int 2014, 85:1112-1122

17. Mulay SR, Kulkarni OP, Rupanagudi KV, Migliorini A, Darisipudi MN, Vilaysane A, Muruve D, Shi Y, Munro F, Liapis H, Anders HJ: Calcium oxalate crystals induce renal inflammation by NLRP3-mediated IL-1 $\beta$ secretion. J Clin Invest 2013, 123:236-246

18. Zaki MH, Boyd KL, Vogel P, Kastan MB, Lamkanfi M, Kanneganti TD: The NLRP3 inflammasome protects against loss of epithelial integrity and mortality during experimental colitis. Immunity 2010, 32:379-391

19. Vilaysane A, Chun J, Seamone ME, Wang W, Chin R, Hirota S, Li Y, Clark SA, Tschopp J, Trpkov K, Hemmelgarn BR, Beck PL, Muruve DA: The NLRP3 inflammasome promotes renal inflammation and contributes to CKD. J Am Soc Nephrol 2010, 21:1732-1744

20. Sutterwala FS, Ogura Y, Szczepanik M, Lara-Tejero M, Lichtenberger GS, Grant EP, Bertin J, Coyle AJ, Galán JE, Askenase PW, Flavell RA: Critical role for NALP3/CIAS1/Cryopyrin in innate and adaptive immunity through its regulation of caspase-1. Immunity 2006, 24:317-327

21. Sauvant C, Schneider R, Holzinger H, Renker S, Wanner C, Gekle M: Implementation of an in vitro model system for investigation of reperfusion damage after renal ischemia. Cell Physiol Biochem 2009, 24:567-576

22. Han WK, Bailly V, Abichandani R, Thadhani R, Bonventre JV: Kidney injury molecule-1 (KIM-1): a novel biomarker for human renal proximal tubule injury. Kidney Int 2002, 62:237-244

23. Paragas N, Qiu A, Zhang Q, Samstein B, Deng SX, SchmidtOtt KM, Viltard M, Yu W, Forster CS, Gong G, Liu Y, Kulkarni R, Mori K, Kalandadze A, Ratner AJ, Devarajan P, Landry DW, D'Agati V, Lin CS, Barasch J: The Ngal reporter mouse detects the response of the kidney to injury in real time. Nat Med 2011, 17: 216-222

24. Sagulenko V, Thygesen SJ, Sester DP, Idris A, Cridland JA, Vajjhala PR, Roberts TL, Schroder K, Vince JE, Hill JM, Silke J, Stacey KJ: AIM2 and NLRP3 inflammasomes activate both apoptotic and pyroptotic death pathways via ASC. Cell Death Differ 2013, 20: $1149-1160$

25. Ricardo SD, van Goor H, Eddy AA: Macrophage diversity in renal injury and repair. J Clin Invest 2008, 118:3522-3530

26. Toback FG: Regeneration after acute tubular necrosis. Kidney Int 1992, 41:226-246

27. Wang W, Wang X, Chun J, Vilaysane A, Clark S, French G, Bracey NA, Trpkov K, Bonni S, Duff HJ, Beck PL, Muruve DA: Inflammasome-independent NLRP3 augments TGF- $\beta$ signaling in kidney epithelium. J Immunol 2013, 190:1239-1249

28. Bonventre J, Yang L: Cellular pathophysiology of ischemic acute kidney injury. J Clin Invest 2011, 121:4210-4221

29. Lorenz G, Darisipudi MN, Anders HJ: Canonical and non-canonical effects of the NLRP3 inflammasome in kidney inflammation and fibrosis. Nephrol Dial Transplant 2014, 29:41-48

30. Kawaida K, Matsumoto K, Shimazu H, Nakamura T: Hepatocyte growth factor prevents acute renal failure and accelerates renal regeneration in mice. Proc Natl Acad Sci U S A 1994, 91:4357-4361

31. Liu Y, Michalopoulos GK, Zarnegar R: Structural and functional characterization of the mouse hepatocyte growth factor gene promoter. J Biol Chem 1994, 269:4152-4160

32. Martinon F, Pétrilli V, Mayor A, Tardivel A, Tschopp J: Gout-associated uric acid crystals activate the NALP3 inflammasome. Nature 2006, 440:237-241

33. Paduch R, Jakubowicz-Gil J, Niedziela P: Hepatocyte growth factor (HGF), heat shock proteins (HSPs) and multidrug resistance protein 
(MRP) expression in co-culture of colon tumor spheroids with normal cells after incubation with interleukin-1beta (IL-1beta) and/or camptothecin (CPT-11). Indian J Exp Biol 2010, 48:354-364

34. Liang CC, Park AY, Guan JL: In vitro scratch assay: a convenient and inexpensive method for analysis of cell migration in vitro. Nat Protoc 2007, 2:329-333

35. Pulskens WP, Butter LM, Teske GJ, Claessen N, Dessing MC, Flavell RA, Sutterwala FS, Florquin S, Leemans JC: Nlrp3 prevents early renal interstitial edema and vascular permeability in unilateral ureteral obstruction. PLoS One 2014, 9:e85775
36. Venkatachalam MA, Griffin KA, Lan R, Geng H, Saikumar P, Bidani AK: Acute kidney injury: a springboard for progression in chronic kidney disease. Am J Physiol Renal Physiol 2010, 298:F1078-F1094

37. Hsu CY: Yes, AKI truly leads to CKD. J Am Soc Nephrol 2012, 23: 967-969

38. Leung KC, Tonelli M, James MT: Chronic kidney disease following acute kidney injury: risk and outcomes. Nat Rev Nephrol 2013, 9:77-85

39. Coca SG, Singanamala S, Parikh CR: Chronic kidney disease after acute kidney injury: a systematic review and meta-analysis. Kidney Int 2012, 81:442-448 